\title{
Existence of chaos for a simple delay difference equation
}

\section{Zongcheng $\mathrm{Li}^{1,2^{*}}$ and Xiaoying Zhu ${ }^{1}$}

${ }^{\text {*Correspondence: }}$ lizongcheng_0905@163.com

'school of Science, Shandong Jianzhu University, Jinan, Shandong 250101, China

${ }^{2}$ College of Control Science and Engineering, Shandong University, Jinan, Shandong 250061, China

\begin{abstract}
In this paper, we study the existence of chaos for a simple delay difference equation. By using the snap-back repeller theory, we prove that the system is chaotic in the sense of both Devaney and Li-Yorke when the parameters of this system satisfy some mild conditions. For illustrating the theoretical result, we give two computer simulations.
\end{abstract}

Keywords: delay; snap-back repeller; chaos in the sense of Devaney; chaos in the sense of Li-Yorke

\section{Introduction}

Time delay appears in many realistic systems with feedback in science and engineering. It affects the behaviors of a dynamical system significantly. The main reason is that it causes a system to be infinite dimensional. For example, Uçar [1] proposed and analyzed a very simple first-order delay differential equation that would admit decaying, oscillatory, and even chaotic solutions. Recently, many researchers studied the following delay differential equation:

$$
\dot{x}(t)=-x(t)+a f(x(t)-b x(t-\tau))
$$

where $a, b$ are real parameters, $\tau>0$ is the delay, and $f: \mathbf{R} \rightarrow \mathbf{R}$ is a real function. Equation (1) is known as a generalization of a neural network. When the function $f(x)$ is taken as $\tanh (x),(1)$ becomes a neural network, which was studied in [2] for the stability characteristics. Particularly, in [3-5], the authors studied the stability and chaos when $f(x)$ and the system parameters $a, b$ satisfy some specific conditions.

It is well known that we can use the discrete analog to study the complex dynamical behaviors of nonlinear differential systems. Some qualitative properties of the corresponding difference equations can provide much useful information for analyzing the properties of the original differential equations. Therefore, it is very important to study properties of difference equations. Many researchers had studied the following delay difference equation:

$$
x(n+1)=c x(n)+g(x(n)-x(n-k))
$$

where $c$ is a parameter, $k$ is a positive integer, and $g: \mathbf{R} \rightarrow \mathbf{R}$ is a map. Equation (2) can be viewed as the Euler discretization of (1) for $b=1$. Such an equation arises from some of

() 2015 Li and Zhu; licensee Springer. This is an Open Access article distributed under the terms of the Creative Commons Attribution License (http://creativecommons.org/licenses/by/4.0), which permits unrestricted use, distribution, and reproduction in any medium, provided the original work is properly credited. 
the earliest mathematical models of the macroeconomic 'trade cycle', and has attracted a great deal of attention; see [6-11], and many references therein. Most of them studied the permanence, boundedness, stability, attractivity, oscillations, and bifurcations.

For the research on chaotic behavior of (2) or discretization of (1), even for some special forms of them, there are few results which are studied with rigorously mathematical proofs. To the best of our knowledge, most of the existing results are studied with the aid of computer simulations. The main goal of this paper is to study the existence of chaos in the discrete analog of (1). However, it is very difficult to study chaos for a general function $f$. In [12], Sprott proposed a simple prototype model with a sinusoidal nonlinearity to study chaos for delay differential equations, which is regarded as one of the simplest chaotic delay differential equations. This motivates us to use the sine function as an example to explore the chaotic behavior in discrete analog of (1). That is, we study the existence of chaos of the following delay difference equation:

$$
x(n+1)=\alpha x(n)+\beta \sin [x(n)-\gamma x(n-k)]
$$

where $\alpha, \beta, \gamma$ are real parameters, and $k$ is a positive integer.

This paper is organized as follows. In Section 2, we give some basic concepts and one lemma. In Section 3, we prove that the delay difference equation is chaotic in the sense of both Devaney and Li-Yorke under some conditions, by using the snap-back repeller theory. Then we give two computer simulations to illustrate the theoretical result. Finally, we conclude this paper in Section 4.

\section{Preliminaries}

Up to now, there is no unified definition of chaos in mathematics. For convenience, we list two definitions of chaos which will be used in this paper.

Definition 1 [13] Let $(X, d)$ be a metric space, $F: X \rightarrow X$ be a map, and $S$ be a set of $X$ with at least two distinct points. Then $S$ is called a scrambled set of $F$ if for any two distinct points $x, y \in S$,

(i) $\liminf _{n \rightarrow \infty} d\left(F^{n}(x), F^{n}(y)\right)=0$;

(ii) $\limsup _{n \rightarrow \infty} d\left(F^{n}(x), F^{n}(y)\right)>0$.

The map $F$ is said to be chaotic in the sense of Li-Yorke if there exists an uncountable scrambled set $S$ of $F$.

Remark 1 The term 'chaos' was first used by Li and Yorke [14] for a map on a compact interval. Following the work of Li and Yorke, Zhou [13] gave the above definition of chaos for a topological dynamical system on a general metric space.

Definition 2 [15] Let $(X, d)$ be a metric space. A map $F: V \subset X \rightarrow V$ is said to be chaotic on $V$ in the sense of Devaney if

(i) the set of the periodic points of $F$ is dense in $V$;

(ii) $F$ is topologically transitive in $V$;

(iii) $F$ has sensitive dependence on initial conditions in $V$.

Remark 2 If $F$ is continuous in $V$, then condition (iii) can be concluded by conditions (i) and (ii), which was shown by Banks et al. [16]. Consequently, condition (iii) is unnecessary 
in Definition 2 if $F$ is continuous in $V$. In [17], Huang and Ye showed that chaos in the sense of Devaney is stronger than that in the sense of Li-Yorke under some conditions.

In the rest of the paper, we use $B_{r}(z)$ and $\bar{B}_{r}(z)$ to denote the open and closed balls of radius $r$ centered at $z \in X$, respectively. The following definitions in [18] are used in this paper.

Definition 3 [18, Definitions 2.1-2.4] Let $(X, d)$ be a metric space and $F: X \rightarrow X$ be a map.

(i) A point $z \in X$ is called an expanding fixed point (or a repeller) of $F$ in $\bar{B}_{r}(z)$ for some constant $r>0$, if $F(z)=z$ and there exists a constant $\lambda>1$ such that

$$
d(F(x), F(y)) \geq \lambda d(x, y) \quad \forall x, y \in \bar{B}_{r}(z) .
$$

The constant $\lambda$ is called an expanding coefficient of $F$ in $\bar{B}_{r}(z)$. Furthermore, $z$ is called a regular expanding fixed point of $F$ in $\bar{B}_{r}(z)$ if $z$ is an interior point of $F\left(B_{r}(z)\right)$. Otherwise, $z$ is called a singular expanding fixed point of $F$ in $\bar{B}_{r}(z)$.

(ii) Assume that $z$ is an expanding fixed point of $F$ in $\bar{B}_{r}(z)$ for some $r>0$. Then $z$ is said to be a snap-back repeller of $F$ if there exists a point $x_{0} \in B_{r}(z)$ with $x_{0} \neq z$ and $F^{m}\left(x_{0}\right)=z$ for some positive integer $m$. Furthermore, $z$ is said to be a nondegenerate snap-back repeller of $F$ if there exist positive constants $\mu$ and $r_{0}<r$ such that $B_{r_{0}}\left(x_{0}\right) \subset B_{r}(z)$ and

$$
d\left(F^{m}(x), F^{m}(y)\right) \geq \mu d(x, y) \quad \forall x, y \in B_{r_{0}}\left(x_{0}\right) .
$$

$z$ is called a regular snap-back repeller of $F$ if $F\left(B_{r}(z)\right)$ is open and there exists a positive constant $\delta_{0}$ such that $B_{\delta_{0}}\left(x_{0}\right) \subset B_{r}(z)$ and for each positive constant $\delta \leq \delta_{0}$, $z$ is an interior point of $F^{m}\left(B_{\delta}\left(x_{0}\right)\right)$. Otherwise, $z$ is called a singular snap-back repeller of $F$.

Remark 3 The concept of snap-back repeller for maps in $\mathbf{R}^{n}$ was introduced by Marotto [19] in 1978. Obviously, Definition 3 is given in general metric spaces, which is an extension of Marotto's definition. In terms of Definition 3, the snap-back repeller given by Marotto [19] is regular and nondegenerate.

Lemma 1 ([20, Theorem 4.4], [21, Theorem 2.1]) Let $F: \mathbf{R}^{n} \rightarrow \mathbf{R}^{n}$ be a map with a fixed point $z \in \mathbf{R}^{n}$. Assume that

(i) $F$ is continuously differentiable in a neighborhood of $z$ and all the eigenvalues of $\mathrm{DF}(z)$ have absolute values larger than 1 , which implies that there exist a positive constant $r$ and a norm $\|\cdot\|$ in $\mathbf{R}^{n}$ such that $F$ is expanding in $\bar{B}_{r}(z)$ in $\|\cdot\|$;

(ii) $z$ is a snap-back repeller of $F$ with $F^{m}\left(x_{0}\right)=z, x_{0} \neq z$, for some $x_{0} \in B_{r}(z)$ and some positive integer $m$. Furthermore, $F$ is continuously differentiable in some neighborhoods of $x_{0}, x_{1}, \ldots, x_{m-1}$, respectively, and $\operatorname{det} \mathrm{DF}\left(x_{j}\right) \neq 0$ for $0 \leq j \leq m-1$, where $x_{j}=F\left(x_{j-1}\right)$ for $1 \leq j \leq m-1$.

Then for each neighborhood $U$ of $z$, there exist a positive integer $k>m$ and $a$ Cantor set $\Lambda \subset U$ such that $F^{k}: \Lambda \rightarrow \Lambda$ is topologically conjugate to the symbolic dynamical system $\sigma: \sum_{2}^{+} \rightarrow \sum_{2}^{+}$. Consequently, there exists a compact and perfect invariant set $V \subset \mathbf{R}^{n}$, 
containing the Cantor set $\Lambda$, such that $F$ is chaotic on $V$ in the sense of Devaney as well as in the sense of Li-Yorke, and has a dense orbit in $V$.

Remark 4 We can easily conclude that $z$ is a regular and nondegenerate snap-back repeller from Lemma 1. Hence, Lemma 1 can be summed as a single word: 'a regular and nondegenerate snap-back repeller in $\mathbf{R}^{n}$ implies chaos in the sense of both Devaney and Li-Yorke.' For more details, one can see [20, 21].

\section{Existence of chaos}

Let $u_{j}(n):=x(n+j-k-1)$ for $1 \leq j \leq k+1$, then (3) is changed into a $(k+1)$-dimensional system on $\mathbf{R}^{k+1}$,

$$
\begin{aligned}
u(n+1) & =\left(\begin{array}{c}
u_{2}(n) \\
u_{3}(n) \\
\vdots \\
u_{k+1}(n) \\
\alpha u_{k+1}(n)+\beta \sin \left[u_{k+1}(n)-\gamma u_{1}(n)\right]
\end{array}\right) \\
: & =F(u(n)),
\end{aligned}
$$

where $u=\left(u_{1}, u_{2}, \ldots, u_{k+1}\right)^{T} \in \mathbf{R}^{k+1}$.

System (4) is called the system induced by (3) in $\mathbf{R}^{k+1}$. It is clear that a solution $\{x(n-k), \ldots, x(n)\}_{n=1}^{\infty}$ of (3) corresponds to a solution $\{u(n)\}_{n=1}^{\infty}$ of system (4), where the initial condition $\{x(-k), \ldots, x(0)\}$ of (3) corresponds to an initial condition $u(0)=$ $\left(u_{1}(0), \ldots, u_{k+1}(0)\right)^{T} \in \mathbf{R}^{k+1}$ of system (4). Hence, we can study the dynamical behavior of (3) by studying that of its induced system (4) in $\mathbf{R}^{k+1}$. So, we call (3) is chaotic in the sense of Devaney (or Li-Yorke) on $V \subset \mathbf{R}^{k+1}$ if its induced system (4) is chaotic in the sense of Devaney (or Li-Yorke) on $V \subset \mathbf{R}^{k+1}$.

Now, we state the main result of this paper as the following theorem.

Theorem 1 There exists a constant $\beta_{0}>0$ such that for arbitrary $\beta$ satisfying $|\beta|>\beta_{0}$ and for some $\gamma$ satisfying

$$
|\gamma|>\frac{1+|\alpha+\beta|}{|\beta|}
$$

system (4), and consequently (3), is chaotic in the sense of both Devaney and Li-Yorke.

Proof Lemma 1 will be used to prove this theorem. Therefore, we only need to show that the map $F$ of system (4) satisfies all the assumptions in Lemma 1.

It is clear that $O:=(0, \ldots, 0)^{T} \in \mathbf{R}^{k+1}$ is always a fixed point of system (4), and other fixed points $P:=\left(x_{0}, \ldots, x_{0}\right)^{T} \in \mathbf{R}^{k+1}$ satisfy

$$
\sin [(1-\gamma) x]=\frac{(1-\alpha) x}{\beta} .
$$

For simplicity, we will only prove that the fixed point $O$ may be a regular and nondegenerate snap-back repeller of the map $F$ when parameters satisfy the conditions in Theorem 1 . 
For simplifying the proof and convenience, $\gamma$ is taken as an integer and satisfies condition (5) throughout the proof.

Firstly, it is to show that $O$ is an expanding fixed point of $F$ in $\mathbf{R}^{k+1}$ under condition (5). It is obvious that $F$ is continuously differentiable in $\mathbf{R}^{k+1}$, and its Jacobian matrix at $O$ is

$$
\mathrm{DF}(O)=\left(\begin{array}{ccccc}
0 & 1 & 0 & \cdots & 0 \\
0 & 0 & 1 & \cdots & 0 \\
. . & . . & . . & \cdots & . . \\
0 & 0 & 0 & \cdots & 1 \\
-\beta \gamma & 0 & 0 & \cdots & \alpha+\beta
\end{array}\right)_{(k+1) \times(k+1)}
$$

The characteristic equation of $\operatorname{DF}(O)$ is

$$
\lambda^{k+1}-(\alpha+\beta) \lambda^{k}+\beta \gamma=0,
$$

from which we obtain the result that all the eigenvalues of $\operatorname{DF}(O)$ have absolute values larger than 1 under condition (5). If it is not true, then there will exist at least an eigenvalue $\lambda_{0}$ of $\operatorname{DF}(O)$ satisfies $\left|\lambda_{0}\right| \leq 1$. So, we can obtain the following contradiction:

$$
\begin{aligned}
1+|\alpha+\beta| & \geq\left|\lambda_{0}^{k+1}\right|+\left|(\alpha+\beta) \lambda_{0}^{k}\right| \\
& \geq\left|\lambda_{0}^{k+1}-(\alpha+\beta) \lambda_{0}^{k}\right|=|-\beta \gamma|>1+|\alpha+\beta| .
\end{aligned}
$$

Therefore, we find that $O$ is an expanding fixed point of $F$ from the first condition of Lemma 1 , that is,

$$
\|F(x)-F(y)\|^{*} \geq \mu\|x-y\|^{*}, \quad \forall x, y \in \bar{B}_{r}(O)
$$

where $r>0$ is a constant, $\|\cdot\|^{*}$ is some norm in $\mathbf{R}^{k+1}$, and $\mu>1$ is an expanding coefficient of $F$ in $\bar{B}_{r}(O)$.

Secondly, one is to prove that $O$ is a snap-back repeller of the map $F$. Let $W \subset \bar{B}_{r}(O)$ be an arbitrary neighborhood of $O$ in $\mathbf{R}^{k+1}$. Then we can obtain a small interval $U \subset \mathbf{R}$ containing 0 such that $\underbrace{U \times U \times \cdots \times U}_{k+1} \subset W$. Now, one is to show that there exists a point $O_{0} \in W$ such that $O_{0} \neq O$ and

$$
F^{k+2}\left(O_{0}\right)=O
$$

When $k=1$, we can achieve a positive constant $\beta_{1}$ such that for arbitrary $|\beta|>\beta_{1}$, there exist two points $x_{1}, x_{2} \in U$ satisfying

$$
\left\{\begin{array}{l}
\beta \sin \left(\gamma x_{2}\right)=-\alpha \pi, \\
\beta \sin \left(x_{2}-\gamma x_{1}\right)=\pi-\alpha x_{2} .
\end{array}\right.
$$

Let $O_{0}=\left(x_{1}, x_{2}\right)^{T} \in \mathbf{R}^{2}$, it follows that $O_{0} \in U \times U \subset W$ with $O_{0} \neq O$ for arbitrary $|\beta|>\beta_{1}$. From (6), we obtain the result that $F\left(O_{0}\right)=\left(x_{2}, \pi\right)^{T}, F^{2}\left(O_{0}\right)=(\pi, 0)^{T}, F^{3}\left(O_{0}\right)=O$. 
When $k>1$, we can also achieve a positive constant $\beta_{2}$ such that for arbitrary $|\beta|>\beta_{2}$, there exist two points $x_{1}, x_{2} \in U$ satisfying

$$
\left\{\begin{array}{l}
\beta \sin \left(\gamma x_{1}\right)=-\pi \\
\beta \sin \left(\gamma x_{2}\right)=-\alpha \pi
\end{array}\right.
$$

Let $O_{0}=\left(x_{1}, x_{2}, 0, \ldots, 0\right)^{T} \in \mathbf{R}^{k+1}$, it also follows that $O_{0} \in \underbrace{U \times U \times \cdots \times U}_{k+1} \subset W$ with $O_{0} \neq$ $O$ for arbitrary $|\beta|>\beta_{2}$. From (7), we also obtain the result that $F\left(O_{0}\right)=\left(x_{2}, 0, \ldots, \pi\right)^{T}$, $F^{j}\left(O_{0}\right)=(0, \ldots, 0, \underbrace{\pi, 0, \ldots, 0}_{j})^{T}$ for $2 \leq j \leq k+1$, and $F^{k+2}\left(O_{0}\right)=O$.

Set $\beta_{0}:=\max \left\{\beta_{1}, \beta_{2}\right\}$. From the above discussion, it follows that for arbitrary $\beta$ satisfying $|\beta|>\beta_{0}$, there exists a point $O_{0} \in W$ satisfying $O_{0} \neq O$ and $F^{k+2}\left(O_{0}\right)=O$. Therefore, $O$ is a snap-back repeller of $F$ for arbitrary $\beta$ satisfying $|\beta|>\beta_{0}$.

Thirdly, one is to prove that for arbitrary $|\beta|>\beta_{0}$, the following holds:

$$
\operatorname{det} \operatorname{DF}\left(O_{j}\right) \neq 0, \quad 0 \leq j \leq k+1
$$

where $O_{j}:=F\left(O_{j-1}\right)$ for $1 \leq j \leq k+1$. The existence of the Jacobian matrices of $F$ at $O_{j}$ $(0 \leq j \leq k+1)$ is because $F$ is continuously differentiable in $\mathbf{R}^{k+1}$.

It is easy to conclude that for arbitrary $u=\left(u_{1}, \ldots, u_{k+1}\right)^{T} \in \mathbf{R}^{k+1}$, the following holds:

$$
\operatorname{det} \operatorname{DF}(u)=(-1)^{k+1} \beta \gamma \cos \left(u_{k+1}-\gamma u_{1}\right)
$$

When $k=1$, it follows that $O_{0}=\left(x_{1}, x_{2}\right)^{T}, O_{1}=\left(x_{2}, \pi\right)^{T}, O_{2}=(\pi, 0)^{T} \in \mathbf{R}^{2}$. From (6), we get $\sin \left(\gamma x_{2}\right) \neq 1$ and $\sin \left(x_{2}-\gamma x_{1}\right) \neq 1$ for arbitrary $|\beta|>\beta_{0}$. Together with (8), we get the following for arbitrary $|\beta|>\beta_{0}$ :

$$
\begin{aligned}
& \operatorname{det} \operatorname{DF}\left(O_{0}\right)=\beta \gamma \cos \left(x_{2}-\gamma x_{1}\right) \neq 0, \\
& \operatorname{det} \operatorname{DF}\left(O_{1}\right)=-\beta \gamma \cos \left(\gamma x_{2}\right) \neq 0, \\
& \operatorname{det} \operatorname{DF}\left(O_{2}\right)=\beta \gamma \cos (\gamma \pi)= \pm \beta \gamma \neq 0 .
\end{aligned}
$$

When $k>1$, it follows that $O_{0}=\left(x_{1}, x_{2}, 0, \ldots, 0\right)^{T}, O_{1}=\left(x_{2}, 0, \ldots, 0, \pi\right)^{T}$, and $O_{j}=$ $(0, \ldots, 0, \underbrace{\pi, 0, \ldots, 0}_{j})^{T} \in \mathbf{R}^{k+1}$ for $2 \leq j \leq k+1$. Similarly, it follows from (7) that $\sin \left(\gamma x_{1}\right) \neq 1$ and $\sin \left(\gamma x_{2}\right) \neq 1$ for arbitrary $|\beta|>\beta_{0}$. Consequently, the following hold for arbitrary $|\beta|>\beta_{0}$ :

$$
\begin{aligned}
& \operatorname{det} \operatorname{DF}\left(O_{0}\right)=(-1)^{k+1} \beta \gamma \cos \left(\gamma x_{1}\right) \neq 0, \\
& \operatorname{det} \operatorname{DF}\left(O_{1}\right)=(-1)^{k} \beta \gamma \cos \left(\gamma x_{2}\right) \neq 0, \\
& \operatorname{det} \operatorname{DF}\left(O_{j}\right)=(-1)^{k+1} \beta \gamma \neq 0, \quad \text { for } 2 \leq j \leq k, \\
& \operatorname{det} \operatorname{DF}\left(O_{k+1}\right)=(-1)^{k+1} \beta \gamma \cos (\gamma \pi)= \pm \beta \gamma \neq 0 .
\end{aligned}
$$

In summary, the map $F$ satisfies all the assumptions in Lemma 1. Consequently, system (4), i.e., (3), is chaotic in the sense of both Devaney and Li-Yorke. This completes the proof. 
Remark 5 For simplifying the proof of Theorem 1, the parameter $\gamma$ is taken as an integer. It should be pointed out that $\gamma$ may be taken as other values such that system (4) is chaotic. In addition, it follows from the above proof that there exists a constant $\beta_{0}>0$ such that for arbitrary $|\beta|>\beta_{0}$, system (4) is chaotic in the sense of both Devaney and Li-Yorke. However, there are few methods to determine the concrete expanding area of a fixed point in the literature. So it is not easy to get the particular value $\beta_{0}$. In practical problems, we can take the parameter $|\beta|$ large enough such that (6) or (7) in the proof of Theorem 1 are satisfied.

For illustrating the theoretical result, we present two computer simulations of system (4), from which we can see that system (4), i.e., (3) indeed has complex dynamical behaviors. The parameters are taken as $\alpha=0.1, \beta=200, \gamma=6, k=1,2$. From the proof of Theorem 1 , we see that $O$ is an expanding fixed point of the map $F$ for $|\gamma|=6>[1+|\alpha+\beta|] /|\beta|=$ 1.0055. It is also easy to obtain the result that there exist two pairs of points $x_{1} \approx-0.1388$, $x_{2} \approx-0.2618$ satisfying (6) when $k=1$, and $x_{1} \approx-0.0026, x_{2} \approx-0.2618$ satisfying (7) when $k>1$. Therefore, $O$ is a regular and nondegenerate snap-back repeller of the map $F$. Two simulation results are given in Figures 1 and 2 for $k=1,2$, which exhibit complex dynamical behaviors of the system.

\section{Conclusion}

In this paper, we rigorously show the existence of chaos in a simple delay difference equation, which illustrates that the discrete analog of system (1) indeed has very complicated dynamical behaviors. By using the snap-back repeller theory, we prove that the system is chaotic in the sense of both Devaney and Li-Yorke when the parameters of this system satisfy some mild conditions. Numerical simulations confirm the theoretical analysis. However, the map $f$ of system (1) is taken as a special function. Therefore, it is very interesting to study the chaotic behavior of system (1) or its discrete analog for a more general form of $f$, which will be our further research.

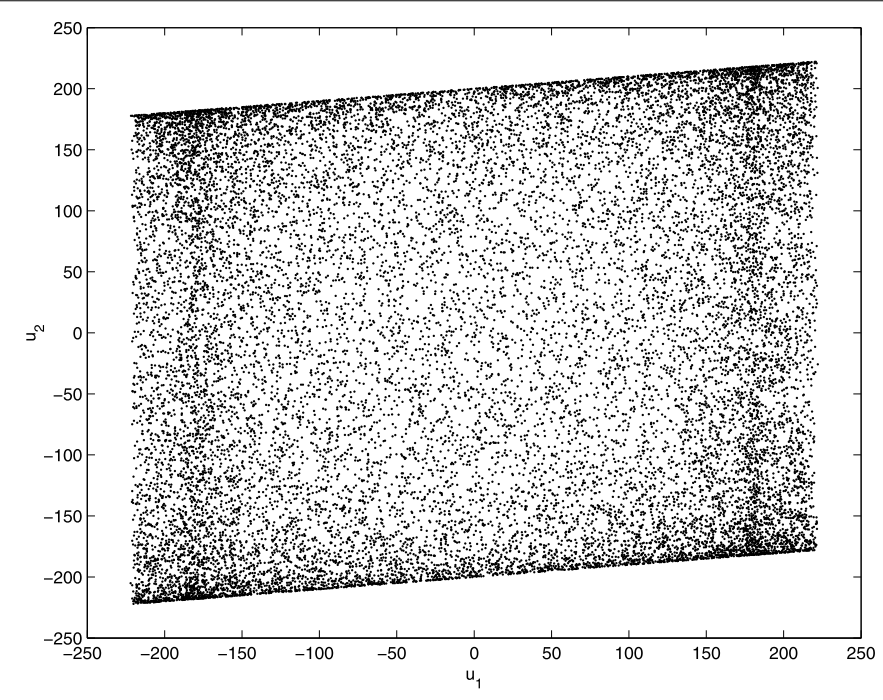

Figure 1 Computer simulation of system (4) for $\alpha=0.1, \beta=200, \gamma=6, k=1$, and $n$ from 0 to 20,000, with the initial condition $u(0)=(0.01,0.01)^{T}$. 


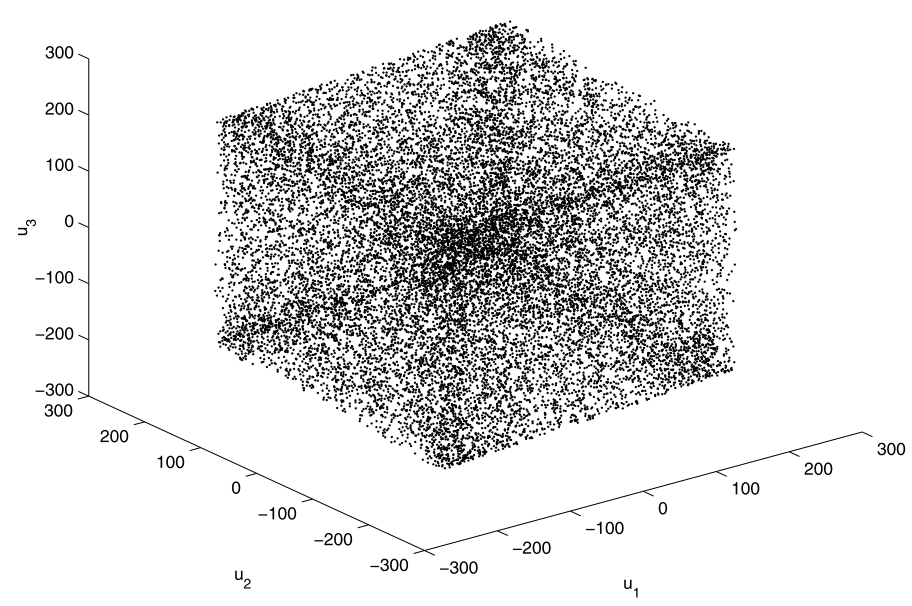

Figure 2 Computer simulation of system (4) for $\alpha=0.1, \beta=200, \gamma=6, k=2$, and $n$ from 0 to 20,000 with the initial condition $u(0)=(0.01,0.01,0.01)^{T}$.

\section{Competing interests}

The authors declare that they have no competing interests.

\section{Authors' contributions}

All authors contributed equally to the writing of this paper. All authors read and approved the final manuscript.

\section{Acknowledgements}

This work was supported by the National Natural Science Foundation of China (Grant 11101246) and the Postdoctoral Science Foundation of China (Grant 2014M561908).

Received: 29 October 2014 Accepted: 13 January 2015 Published online: 11 February 2015

\section{References}

1. Uçar, A: A prototype model for chaos studies. Int. J. Eng. Sci. 40, 251-258 (2002)

2. Gopalsamy, K, Leung, IKC: Convergence under dynamical thresholds with delays. IEEE Trans. Neural Netw. 8, 341-348 (1997)

3. Liao, XF, Wong, KW, Leung, CS, Wu, ZF: Hopf bifurcation and chaos in a single delayed neuron equation with non-monotonic activation function. Chaos Solitons Fractals 12, 1535-1547 (2001)

4. Zhou, SB, Liao, XF, Yu, JB: Chaotic behavior in a simplest neuron model with discrete time delay. J. Electron. Inf. Technol. 24, 1341-1345 (2002)

5. Zhou, SB, Li, H, Wu, ZF: Stability and chaos of a neural network with uncertain time delays. Lect. Notes Comput. Sci. 3496, 340-345 (2005)

6. Hickas, JR: A Contribution to the Theory of Trade Cycle. Clarendon Press, Oxford (1965)

7. Blatt, JM: Dynamic Economic Systems: A Post-Keynesian Approach. M. E. Sharpe, New York (1983)

8. Sedaghat, $\mathrm{H}$ : A class of nonlinear second order difference equations from macroeconomics. Nonlinear Anal. 29 593-603 (1997)

9. Dai, BX, Zhang, N: Stability and global attractivity for a class of nonlinear delay difference equations. Discrete Dyn. Nat. Soc. 2005, 227-234 (2005)

10. Li, SP, Zhang, WN: Bifurcations in a second-order difference equation from macroeconomics. J. Differ. Equ. Appl. 14, 91-104 (2008)

11. El-Morshedy, HA: On the global attractivity and oscillations in a class of second-order difference equations from macroeconomics. J. Differ. Equ. Appl. 17, 1643-1650 (2011)

12. Sprott, JC: A simple chaotic delay differential equation. Phys. Lett. A 366, 397-402 (2007)

13. Zhou, ZL: Symbolic Dynamics. Shanghai Scientific and Technological Education Publishing House, Shanghai (1997)

14. Li, TY, Yorke, JA: Period three implies chaos. Am. Math. Mon. 82, 985-992 (1975)

15. Devaney, RL: An Introduction to Chaotic Dynamical Systems. Addison-Wesley, New York (1987)

16. Banks, J, Brooks, J, Cairns, G, Davis, G, Stacey, P: On Devaney's definition of chaos. Am. Math. Mon. 99, $332-334$ (1992)

17. Huang, W, Ye, XD: Devaney's chaos or 2-scattering implies Li-Yorke's chaos. Topol. Appl. 117, 259-272 (2002)

18. Shi, YM, Chen, GR: Chaos of discrete dynamical systems in complete metric spaces. Chaos Solitons Fractals 22 , 555-571 (2004)

19. Marotto, FR: Snap-back repellers imply chaos in $R^{n}$. J. Math. Anal. Appl. 63, 199-223 (1978)

20. Shi, YM, Chen, GR: Discrete chaos in Banach spaces. Sci. China Ser. A 34, 595-609 (2004). English version: 48, 222-238 (2005)

21. Shi, YM, Yu, P, Chen, GR: Chaotification of dynamical systems in Banach spaces. Int. J. Bifurc. Chaos 16, 2615-2636 (2006) 